\title{
A Resilience Approach to Symbiosis Networks of Ecoindustrial Parks Based on Cascading Failure Model
}

\author{
Yu Zeng, ${ }^{1,2}$ Renbin Xiao, ${ }^{1}$ and Xiangmei $\mathrm{Li}^{3}$ \\ ${ }^{1}$ Institute of Systems Engineering, Huazhong University of Science and Technology, Wuhan 430074, China \\ ${ }^{2}$ School of Science, Hubei University of Technology, Wuhan 430068, China \\ ${ }^{3}$ Wenhua College, Huazhong University of Science and Technology, Wuhan 430074, Hubei, China \\ Correspondence should be addressed to Renbin Xiao; rbxiao@ hust.edu.cn
}

Received 3 April 2013; Accepted 19 May 2013

Academic Editor: Tingwen Huang

Copyright (C) 2013 Yu Zeng et al. This is an open access article distributed under the Creative Commons Attribution License, which permits unrestricted use, distribution, and reproduction in any medium, provided the original work is properly cited.

\begin{abstract}
Evaluation and improvement of resilience in ecoindustrial parks have been the pressing issues to be addressed in the study of safety. In this paper, eco-industrial systems are extracted as symbiosis networks by using social network analysis first. We then construct a novel cascading failure model and propose an evaluation method of node importance according to the features of symbiosis networks of eco-industrial parks. Based on the cascading model, an effective new method, that is, the critical threshold, is put forward to quantitatively assess the resilience of symbiosis networks of eco-industrial parks. Some theoretical analysis is furthermore provided to the critical threshold. Finally, we take Jinjie eco-industrial system in Shanxi Province of China as a case to investigate its resilience. The key potential nodes are identified by using our model. We also find the respective relation among the resilience of symbiosis networks and the parameters in our cascading model. Theoretical analysis results and numerical simulations both show the optimal value of the tunable load parameter with which the strongest resilience level against cascading failures can be attained in symbiosis networks of eco-industrial parks.
\end{abstract}

\section{Introduction}

Complex systems are everywhere in the nature [1-5]. Their Security could have a big impact on the economic development and social stability, so it has attracted a great deal of attention [6-9]. Complex systems can be described as all kinds of networks. Complex networks have become an indispensable part of our life, such as social ecological networks, transportation networks, and power grid networks. Particularly, cascading failure phenomenon of complex networks has been the focus of many recent studies, for the reason that cascading failures are common phenomenon in real-life systems and can cause huge damages to our life and industry development [10-13]. Symbiosis networks of ecoindustrial parks (abbreviated as EIPs hereafter) are also typical networks including cascading phenomenon. In EIPs, the products produced by one enterprise could be the nutrients of other enterprises. Through this kind of cooperation, they form the industrial symbiosis networks, where there exist fairly close industrial symbiosis relations between or among products produced by different enterprises. In the symbiosis network of an EIP, when an upstream enterprise or link failure emerges, the insufficiency of supplying goods to the downstream enterprises will happen. Even though the failure emerges very locally in the ecoindustrial system, it will also quickly spread like a plague to large areas, causing serious damage to the whole system and even resulting in the global collapse. In this way, the entire symbiosis network can be largely affected due to cascading failures. Therefore, it is very necessary to study cascading failures in symbiosis networks of EIPs.

Recently, a plenty of EIPs are constructed in many places, such as Denmark, America, Japan, and Austria. In China, Guangdong Nanhai, Hunan Changsha, Xinjiang Shihezi, Shanghai Wujing, and others have also constructed some ecoindustrial parks. With the development of industrial ecology, the EIP is developing as a brand new research field [14-16]. The research on the complexity of ecoindustrial 
systems has become popular and gradually made a breakthrough. Graph theory was adopted to introduce the flow of materials or energy in Choctaw ecoindustrial park first [17]. Furthermore, Sterr and Ott [18] introduced the flow path of materials or energy in the industrial region network of Rhine-Neckar and made clear the relationship between the enterprise inside the network and that outside the network. Graedel et al. [19] used statistical indexes of the food web from natural ecosystems for reference in his evaluations of EIPs. They assessed the business symbiosis in industrial systems through species richness and connectedness. Based on the work, the potential contribution of industrial symbiosis networks to environmental innovation was explored [16]. In order to measure the complexity characteristics of industrial symbiosis networks, network complexity of two cases, the Kalundborg system in Denmark and the Gongyi system in China were studied. The features of industrial symbiosis networks from the aspects of scale, aggregation, connectedness, complexity, and node influences were analyzed [20]. Different from these methods, a methodology for translating ecological quantitative analysis techniques to an industrial context was proposed. The methodology is demonstrated in the case of Burnside industrial park using the concepts of connectance and diversity. The demonstrated techniques can potentially aid in gaining an understanding of industrial symbiosis [21]. In addition, Posch [22] investigated whether industrial recycling networks or industrial symbiosis projects can be used as a starting point for broader intercompany cooperation for sustainable development.

Although much research on EIPs focuses on the exchange analysis of material or energy, little work has been published on the topology structure of symbiosis networks of EIPs. Especially to date, most researchers have investigated the cascading phenomenon in power grid networks and traffic networks $[23,24]$. Little attention has been paid to the cascading phenomenon in symbiosis networks of EIPs uptil now. Facing the upsurge of ecoindustrial park construction, a set of scientific appraise index systems needs to be established. A challenging problem in industrial ecology is whether industrial symbiosis systems bear complexity features if the systems are described only from their mass or energy interlinkages. Moreover, various members play various roles in symbiosis networks of EIPs. If a critical component fail, the failure will spread very quickly, and it could trigger a more serious damage to symbiosis networks of EIPs due to cascading failures.

Taking the above existing problems into account, we use complex network theory to go into the interior of symbiosis networks and analyze the resilience against cascading failures in symbiosis networks of EIPs in this paper. We abstract EIPs as symbiosis networks and construct a novel cascading model for symbiosis network according to the EIP's characteristics. We use the ecological degree in EIPs for reference and raise a measure to quantitatively evaluate the power and status of nodes. Based on the cascading model, an effective new concept, that is, the critical threshold is put forward to quantitatively assess the resilience of symbiosis networks of EIPs. We take Jinjie ecoindustrial system in Shanxi Province of China as a case to investigate its resilience. Through our proposed model, some potential critical nodes which are sensitive to the ecological degree and the global connectivity of the symbiosis network but not so important intuitively are found. The respective relation among the resilience of symbiosis networks and the parameters is also found in our cascading model. Moreover, according to theoretical analysis results and numerical simulations, we obtain an interesting result; that is, when the value of the tunable load parameter is 1 , the symbiosis network displays the strongest resilience level against cascading failures. These results in this paper may be very helpful for symbiosis networks of EIPs to enhance the resilience against cascading failures. Our work also may have practical implications for promoting the construction of EIPs.

The remainder of this paper is organised as follows. Symbiosis networks of ecoindustrial parks are established with social network analysis in Section 2. According to the construction method, an illustrative example is also given in this section. Section 3 proposes the cascading failure model and an evaluation method of node importance according to the features of ecoindustrial parks. Section 4 gives some theoretical analysis and numerical simulations to our model. Finally, Section 5 concludes with some discussions.

\section{Symbiosis Network Construction of Ecoindustrial Parks}

As one direct practical form of industrial ecology, ecoindustrial parks are becoming important research contents of industrial ecology. The ecoindustrial park (EIP) is defined as an industrial park in which enterprises cooperate with each other by using each other's by-products and wastes; through this kind of cooperation, they form a symbiosis network. Network analysis is a methodology to study objects as a part of a larger system, so it is a brand new feeling to study EIPs through this method. Here, the symbiosis network of an EIP is a network that consists of nodes (components) and the connections between them. In order to investigate the status of all components in EIPs and the resilience of symbiosis networks of EIPs, we will extract ecoindustrial parks as symbiosis networks with social network analysis. The detailed method of building symbiosis network models is as follows.

(1) Nodes and edges of symbiosis networks of EIPs: We confirmed the members of the parks first. The area products, by-products, wastes, and materials are the foundation nodes. Using the administrative boundary as the network boundary, we add some members outside the parks who exchange resources with members within the parks as the supplement nodes. Then we investigate the flow between members, such as exchanges of products, by-products, wastes, and materials. When there is a flow of matter or energy between any two members in the parks, we say that there is an edge between them.

(2) Adjacency matrices of symbiosis networks of EIPs: on the basis of confirmed nodes and edges among members, we establish a relational data collection. We use the directive dichotomous assessment system to 
judge whether the relation exists or not and call the system the adjacency matrix. The adjacency matrix is a square matrix in which if there is one relation between longitudinal and transverse members, the crossover box should be filled with 1 , that is, $a_{i j}=1$, otherwise 0; that is, $a_{i j}=0$. Obviously, if the matrix represents an undirected graph, then it is symmetric as $a_{i j}=a_{j i}$.

(3) Symbiosis network models of EIPs: when one node fails, it not only could lead to the insufficient supply of matter to the downstream enterprises but also decrease the matter outflow of the upstream enterprises at the inverse direction. Members in symbiosis networks cooperate with each other. So we regard symbiosis networks of EIPs as undirected networks. Furthermore, considering that the exchange of data of matter between nodes is difficult to obtain and the measurement standard could also not be unified, we therefore extract ecoindustrial parks as undirected and unweighted symbiosis networks which consist of nodes and edges.

Here, we take the Jinjie ecoindustrial park in Shanxi Province of China as a case to build a symbiosis network model. The EIP consists of four industrial systems: the industrial system of coal power, the industrial system of coal chemical, the industrial system of salt chemical, and the industrial system of glass building materials. This ecoindustrial system is designed to transform resources in situ, develop comprehensive utilization of materials, and produce minimal emissions. A lot of exchanges of matter or energy exist in Jinjie ecoindustrial system, and it is a relatively complex industrial symbiosis network. We choose products, by-products, wastes, and materials from a certain scale of enterprises whose annual output values are more than 2 million yuan as 75 nodes of the symbiosis network. These nodes come from four different industrial systems, respectively, including outsourcing coal, semicoke, coal tar, coal gangue, coal mine, methyl alcohol, solid sulfur, condensed water, salt, polyvinyl chloride, caustic soda, methane oxides, quartz sand, industrial silicon, glass, glass bricks, waste heat, waste water, waste residues, and waste gas. By collecting data and investigating the interrelation between members of Jinjie ecoindustrial park, we obtain the adjacency matrix. According to the adjacency matrix of the symbiosis network, we could obtain the symbiosis network model of Jinjie ecoindustrial park under UCINET 6.0, as is shown in Figure 1.

\section{Model of Cascading Failure in Symbiosis Networks of Ecoindustrial Parks}

In EIPs, the products produced by one enterprise could be the nutrients of other enterprises. Components in EIPs cooperate with each other. If an upstream enterprise or link failure emerges, it not only could lead to the insufficient supply of matter to the downstream enterprises but also decrease the matter outflow of the upstream enterprises at the inverse direction. The fairly close industrial symbiosis relations exist in symbiosis networks. In order to show the cascading phenomenon in symbiosis networks of ecoindustrial parks, we will focus on cascades triggered by the removal of a component. For the symbiosis network of an EIP, initially the network is in a stationary state in which the load of each node is smaller than its capacity. The production system with the EIP maintains the normal operationsm but the removal of a node will change the load balance and lead to the load redistribution over other nodes. Once the capacity of these nodes is insufficient to handle the extra load, this must be induce the node further breaking, triggering a cascade of overload failures and eventually a large drop in the performance of the network. Therefore, the removal of one node can cause serious consequences. In the following, we will construct a cascading failure model according to the characteristics of EIPs.

3.1. A New Cascading Failure Load Model. We define a network $G=(V, E)$ by a nonempty set of nodes $V=$ $\{1,2, \ldots, N\}$ and a nonempty set of edges $E=\{i j \mid i, j \in V\}$. In most of the previous cascade failure models, the load on a node (or an edge) was generally estimated by its degree or its betweenness $[25,26]$. The degree method is inferior owing to its consideration of only a single node degree. Thus, it will lose much information in many actual applications. The betweenness method is not beneficial to its consideration of topological information for the whole network. In symbiosis networks of EIPs, considering that the load of a component is associated with its degree and the degrees of its neighbor components due to the close relation between supply and demand, we assume that the initial load $L_{i}$ of node $i$ being dependent on the degree of node $i$ and the degrees of its neighbor nodes, the expression of which is defined as follows:

$$
L_{i}=k_{i}^{\beta}+\left(\sum_{m \in H_{i}} k_{m}\right)^{\beta}
$$

where $k_{i}$ and $H_{i}$ are the degree of the node $i$ and the set of its neighbor nodes, respectively, and $\beta$ is the tunable parameter which controls the strength of the initial load of a node.

We know that each node of symbiosis networks has a capacity which is the largest load that the node can handle. In symbiosis networks of EIPs, the load capacity of each node is severely limited by cost. Therefore, we assume that the load capacity $C_{i}$ of node $i$ is proportional to its initial load; that is,

$$
C_{i}=(1+\alpha) L_{i}(0), \quad i=1,2, \ldots, N,
$$

where $\alpha>0$ is the tolerance parameter which characterizes the resistance to the attacks. For the symbiosis network of an EIP, the tolerance parameter $\alpha$ also reflects the construction cost of the EIP.

After node $i$ is removed from the network, its load will be redistributed to its neighbor node $j$. We assume that 


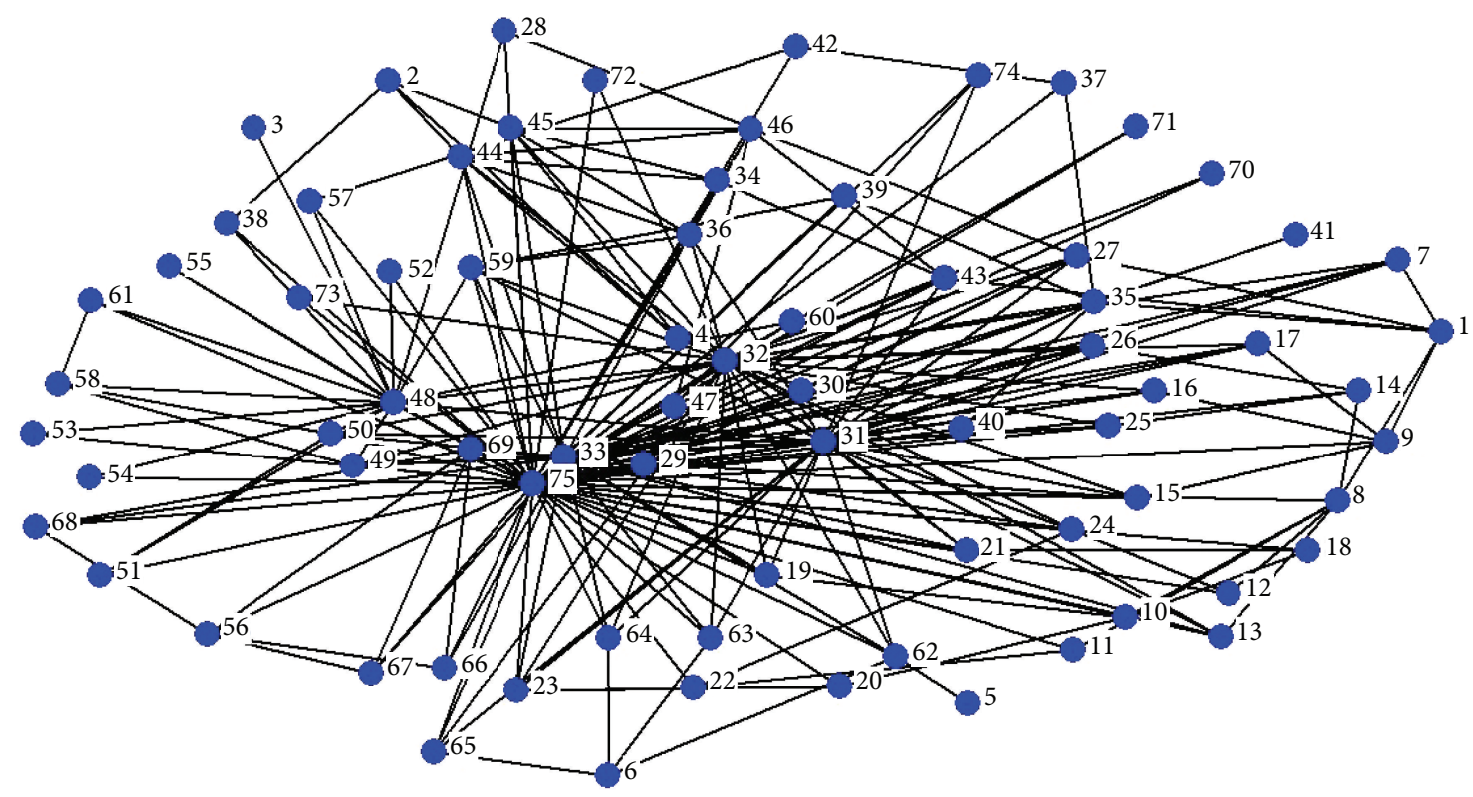

FIGURE 1: The symbiosis network representation of Jinjie ecoindustrial park.

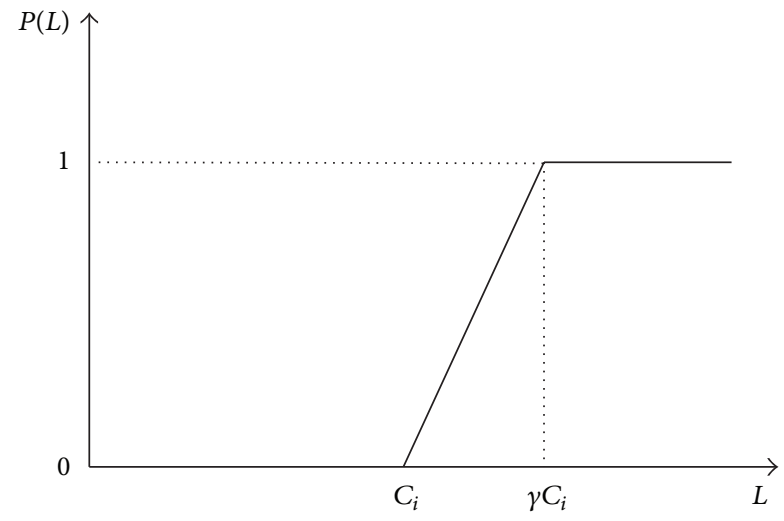

Figure 2: The removal probability $P(L)$ of node $i$, at which load $L$ lasts for time $T$.

the additional load $\Delta L_{i j}$ received by the neighbor node $j$ is proportional to its initial load; that is,

$$
\begin{aligned}
\Delta L_{i j} & =\frac{L_{j}}{\sum_{f \in H_{i}} L_{f}} \times L_{i} \\
& =\frac{k_{j}{ }^{\beta}+\left(\sum_{f \in H_{j}} k_{f}\right)^{\beta}}{\sum_{f \in H_{i}}\left[k_{f}{ }^{\beta}+\left(\sum_{l \in H_{f}} k_{l}\right)^{\beta}\right]} \times\left[k_{i}{ }^{\beta}+\left(\sum_{f \in H_{i}} k_{f}\right)^{\beta}\right] .
\end{aligned}
$$

The method of immediate removal of an instantaneously overloaded node is widely adopted in most of the previous cascade failure models $[25,26]$. This method does not consider the overloaded node removal mechanism in real life. According to the actual situation of EIPs, a certain quantity of instantaneous node overload is permissible and the failure of a node is mainly caused by the accumulative effect of overload, especially for EIPs. In symbiosis networks of EIPs, the insufficient supply of goods induced by a small occasional incident usually has no distinct effect on the normal operation of the whole symbiosis networks. It is because that there exists a certain monitoring and control system in such networks. Once the node overload is detected, some effective measures are taken within the response time to decrease the capacity constraint violation and even can make the node load less than its capacity. In other words, the overloaded node is not necessarily removed. Therefore, we introduce a new method of the overloaded node removal to reflect ecoindustrial systems.

Suppose that $P(L)$ is the removal probability of a node, at which load $L$ lasts for a period of time $T$, as shown in Figure 2. The probability density of $P(L)$ obeys uniform distribution of the interval $[0, T]$. The value of $T$ has some relation to the response time of industrial symbiosis systems. The detailed expression of $P(L)$ is as follows:

$$
P(L)= \begin{cases}0, & L \leq C_{i}, \\ \frac{L-C_{i}}{(\gamma-1) C_{i}}, & C_{i}<L<\gamma C_{i} \\ 1, & L \geq \gamma C_{i}\end{cases}
$$

where the parameter $\gamma(\gamma>1)$ may be different for various symbiosis networks and the value of $\gamma$ determines to a certain extent the protection of those overload nodes. Obviously, the bigger the value of $\gamma$, the higher the cost of avoiding cascading failures. In (5), the three cases correspond to three states of nodes: normal state, overload state, and failure state. 
In the simulations, the following iterative rule is adopted at each time $t$ :

$$
\begin{array}{r}
h_{i}(t)= \begin{cases}h_{i}(t-1)+\frac{P\left(L_{i}(t)\right)}{T}, & L_{i}(t)>C_{i}, \\
0, & L_{i}(t) \leq C_{i},\end{cases} \\
i=1,2, \ldots, N,
\end{array}
$$

where $h_{i}(t)$ is the removal probability of node $i$ at time $t$ and $L_{i}(t)$ is the load of node $i$ at time $t$.

We obtain $P\left(L_{i}(t)\right)$ according to Figure 2 and (5) and derive the malfunction probability $h_{i}(t)$ from (6). According to the above process, the loads of all nodes are calculated at each time after an initial removal of a node induced by a malfunction. We then determine the nodes which will be removed from the network by comparing the malfunction probability and a random $\delta \in(0,1)$. The cascading failure stops until the loads of all nodes are not larger than their corresponding capacity.

\subsection{Node Importance Evaluation Based on Cascading Model.} In the symbiosis network of an EIP, various nodes have different effects on the operation and management of ecoindustrial system, especially for the ecological degree which is employed to measure the relation of exchanging by-products and wastes. The failures of critical nodes are more likely to cause the collapse of the whole ecoindustrial system due to cascading failures. If we can provide priority protection for those critical nodes in advance, the resilience and security of symbiosis networks of EIPs can be improved. To better understand the cascading failure characteristics, in this section, we will discuss and propose a new evaluation method of node importance considering cascading failure for symbiosis networks of EIPs.

After the cascading process is induced by removing a node, the total number of residual nodes is called the network area. In order to find a suitable evaluation method of node importance, we define the following indicator by adopting the network area first:

$$
R_{i}=1-\frac{N_{i}^{\prime}}{N}
$$

where $N$ is the number of all nodes before cascading failures and $N_{i}^{\prime}$ is the number of the nodes which can maintain the normal operations after cascading failures induced by removing node $i$ in the whole symbiosis network. Obviously, the value of indicator $R_{i}$ reflects the damage degree of node $i$ to the global connectivity of symbiosis networks.

Then, the set $V$ of all nodes in the symbiosis network is divided into two categories $V=V_{1} \cup V_{2}$, where the set $V_{2}=\left\{n_{1}, n_{2}, \ldots, n_{N_{e}}\right\}$ consists of waste nodes and by-product nodes which are all called ecological nodes, and the set $V_{1}=$ $\left\{m_{1}, m_{2}, \ldots, m_{N_{p}}\right\}$ consists of all other nodes which are called product nodes. The number of all nodes is $N=N_{e}+N_{p}$. We propose a parameter which is called the rate of ecological connectance $R_{e}$. It is defined as follows:

$$
R_{e}=\frac{\sum_{a \in V_{2}} k_{a}}{N(N-1) / 2},
$$

where $k_{a}$ is the degree of node $a$ and the summation is over the degrees of all ecological nodes. From (8), the higher the value of $R_{e}$ is, the more the flow of the reciprocal utilization of by-products and wastes within EIP is.

We know that the development aim of EIPs is maximum utilization of matter and energy. To reflect the feature of symbiosis networks of EIPs which is different from other networks, now we define another indicator which can be used to characterize the node's impact on the ecological degree of symbiosis networks as follows:

$$
I_{i}=1-\frac{R_{e}^{\prime}}{R_{e}}=1-\frac{\sum_{b \in V_{2} \cap V_{i}^{\prime}} k_{b}}{\sum_{a \in V_{2}} k_{a}},
$$

where $R_{e}$ and $R_{e}^{\prime}$ are the rates of ecological connectance before and after cascading failures in symbiosis networks of EIPs, respectively. $V_{i}^{\prime}$ is the set of residual nodes under the normal operations in the whole network after cascading breakdowns induced by removing node $i$. That is, the numerator of the fraction is the summation over the degree of all residual ecological nodes after cascading breakdowns caused by the removal of node $i$, and the denominator is the summation over the degree of all ecological nodes before cascading breakdowns. The indicator $I_{i}$ characterizes the damage degree of node $i$ to the rate of ecological connectance. The higher the value of indicator $I_{i}$, the greater the impact of node $i$ on the ecological degree of EIPs.

In order to compare indicators $R_{i}$ and $I_{i}$, we apply the two methods to the symbiosis network of Jinjie ecoindustrial park in Section 2 and analyze its cascading failure characteristics. In the symbiosis network of Jinjie ecoindustrial park, the set $V_{2}$ has 14 nodes, such as waste water, waste residue, and waste gas, and the set $V_{1}$ consists of other 61 nodes, such as outsourcing coal, salt, and quartz sand. In the simulations, we choose $\alpha=0.2, \beta=0.2$, and $\gamma=0.3$. Every node in the network is removed one by one, and the corresponding results are calculated, for example, removing the node $i$ and calculating $R_{i}$ and $I_{i}$ by (7) and (9) after the cascading process is over.

Figure 3 shows the values of two indicators for each node. We see that the change in $I_{i}$ is more obvious than $R_{i}$. For example, the values of indicators $R_{6}, R_{7}$ are the same. It indicates that the removals of node 6 and node 7 lead to the same damage degree to the global connectivity of the symbiosis network. However, the difference in $I_{6}$ and $I_{7}$ illustrates that the two nodes' removal has a different impact on the ecological degree of the symbiosis network. Similarly, although the values of $R_{74}$ and $R_{75}$ are same, the removals of node 74 and node 75 cause different damage on ecological degree according to the values of $I_{74}$ and $I_{75}$. The same cases also happen in other nodes. Furthermore, we also find that the removals of nodes $1,2,6,7,9-28,33-39,42-46,48-51$, $53,56-59,61-68,74$, and 75 do not cause cascading failures. It implies that their importance is relatively weak. Despite this, the removals of these nodes result in different impacts on the ecological degree according to indicator $I_{i}$. So we can declare that indicator $I_{i}$ is more helpful in finding out the potential key nodes than indicator $R_{i}$. In other words, indicator $I_{i}$ is a more suitable measure to evaluate the node importance and 


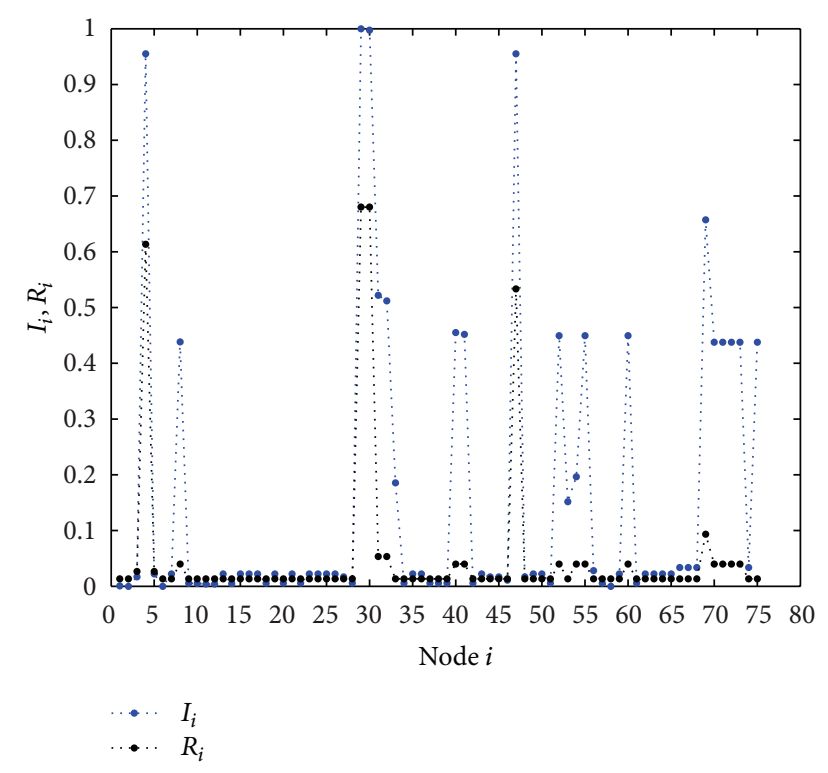

FIgURE 3: Comparison of two indicators for each node.

reflect the status and role of nodes for symbiosis networks of EIPs. It is because that this method can help us to find some potential critical nodes which are sensitive to the ecological degree of symbiosis networks and the efficiency but not so important intuitively.

With the above analysis, we choose indicator $I_{i}$ as the measurement of node importance. All nodes are sorted according to the descending order of the values of indicator $I_{i}$. Table 1 represents the detailed cascading failure processes induced by the nodes that take the top 5 places in ranking. The result shows that node 29 is the most important node in the symbiosis network of Jinjie ecoindustrial park because the failure of node 29 (outsourcing coal) leads to the collapse of the whole symbiosis network. We also find that node 30 (waste water), node 4 (salt), node 47 (waste residue), node 69 (quartz sand), and node 31 (waste heat) rank 2nd, $3 \mathrm{rd}$, 4th, and 5th respectively. The removals of these nodes result in great changes in the ecological degree. They also occupy a very important status. The simulation results are reasonably consistent with the actual situation. Therefore, in the operation and management of Jinjie ecoindustrial park, we should pay more attention to the enterprises in advance that product or deal with these critical nodes, such as waster water, salt, waste residue, waste heat, quartz sand, and especially outsourcing coal.

In addition, we also find that the highest degree or load node is not necessarily one of the most important nodes, and the low degree or load node may also occupy an important status. For instance, the load and the degree of node 30 are both low. Its removal leads to neighbor nodes 27, 75 malfunction first. This triggers a cascade of overload failures and eventually results in the whole network almost collapsing. As a result, there is a large drop in the ecological degree of the whole network after the cascading process is over. It is shown that node 30 is actually a potential key node. Therefore, the high or low ecological degree does not absolutely depend on the node degree or node load. Especially for symbiosis networks of EIPs, the exchange and recycling of by-products and wastes is the most essential and direct factor.

By applying our model to the symbiosis network of Jinjie ecoindustrial park, one can see that our new evaluation method of node importance characterizes the status of node well. This measure can help us to identify some potential critical nodes in symbiosis networks of EIPs. Through protecting these nodes in the operation and management in advance and making an effort to strengthen their construction, we can achieve the comprehensive utilization of matter and energy between components and even achieve the optimal resource allocation. The ecological construction will be promoted systematically with the step-by-step breakthrough method. The cost of pollution treatment not only will be decreased, but also the considerable economic and social benefits will be obtained. Thus, the resilience and security of symbiosis networks of EIPs are improved, especially at the same time the development of EIPs will be promoted.

\section{Resilience Analysis for Symbiosis Networks of EIPs on Model}

It is known that the safety of EIPs severely affects the economic development and the social stability. Therefore, it is very necessary to evaluate and improve resilience of symbiosis networks of EIPs. In this section, we will go into the interior of symbiosis networks and propose the concept of critical threshold to characterize the resilience of symbiosis networks against cascading failures by using our cascading model.

4.1. A Quantitative Measurement of Resilience for Symbiosis Networks of EIPs Based on Cascading Model. CF $F_{i}$ denotes the avalanche size, that is, the number of broken nodes induced by removing node $i$. Obviously, $0 \leq C F_{i} \leq N-1$. We remove every node in a network one by one and calculate the corresponding results, for example, removing the node $i$ and calculating $C F_{i}$ after the cascading process is over. Then, the normalized avalanche size is defined as follows:

$$
C F^{*}=\frac{\sum_{i \in V} C F_{i}}{N(N-1)}
$$

where $V$ represents the set of all nodes in the symbiosis network of an EIP.

From the proposed model, the load capacity $C_{i}$ of the node $i$ is proportional to its initial load and precisely $C_{i}=$ $(1+\alpha) L_{i}(0), i=1,2, \ldots, N$. Given a value of the tunable parameter $\beta$, when the value of the tolerance parameter $\alpha$ is sufficiently small, we can imagine that it is easy for the whole network to fully collapse in the case of an arbitrary node failure because the capacity of each node is limited. On the other hand, for sufficiently large $\alpha$, since all nodes have the larger extra capacities to tolerate the load, no cascading failure occurs and the network maintains its normal and efficient functioning, but at the same time it will need greater network cost because the load capacity of each node is limited by the 
TABLE 1: The cascading failure process and node weight ranking of the top 5 places.

\begin{tabular}{|c|c|c|c|c|c|}
\hline Node number & Cascading failure process & $R$ & $I$ & Ranking & Weight \\
\hline 29 & $\begin{array}{c}\{27,75\} \rightarrow\{28,30-32\} \rightarrow\{35,43\} \rightarrow\{48-50,59,62-65\} \rightarrow\{1,5,6,33,34,36,37 \\
39-41,46,51,58\} \rightarrow\{7-9,42,44,45,47,61,66-69\} \rightarrow\{2,4,15-17,56,57 \\
74\} \rightarrow\{38\}\end{array}$ & 0.6800 & 1 & 1 & 0.0843 \\
\hline 30 & $\begin{array}{c}\{27,75\} \rightarrow\{28,29,31,32\} \rightarrow\{35,43,48-50,59,62-65\} \rightarrow\{1,5,6,33,34,36,37 \\
39,40,41,46,51,58\} \rightarrow\{7-9,42,44,45,47,61,66-69\} \\
\rightarrow\{2,4,15-17,56,57 \\
74\} \rightarrow\{38\}\end{array}$ & 0.6800 & 0.9976 & 2 & 0.0841 \\
\hline 4 & $\begin{aligned}\{2,45\} & \rightarrow\{38,44\} \rightarrow\{32-34,46,48,75\} \\
62-65\} \rightarrow\{1,5,6,31,37,39,40,41,51,58,74\} & \rightarrow\{7,8,36,43,47,49,50,59 \\
28-30,57\} & \end{aligned}$ & 0.6133 & 0.9551 & 3 & 0.0805 \\
\hline 47 & $\begin{aligned}\{46,74\} & \rightarrow\{31,75\} \rightarrow\{36,43\} \rightarrow\{1,32-34,44,45,59\} \rightarrow\{2,4,27,35,39,48-50 \\
& 62-65\} \rightarrow\{5,6,28-30,37,38,40,41,51,58\} \rightarrow\{42,61\} \rightarrow\{57\}\end{aligned}$ & 0.5333 & 0.9396 & 4 & 0.0786 \\
\hline 69 & $\{33,56,75\} \rightarrow\{66,67,68\} \rightarrow\{56\}$ & 0.1067 & 0.6573 & 5 & 0.0554 \\
\hline
\end{tabular}

cost of the node. Thus, with the increase of the parameter $\alpha$, there should be some crossover behavior of the system from large scale breakdown to no breakdown, going through small scale ones. Therefore, we use the crossover behavior to quantify the network resilience, that is, the critical threshold $\alpha_{c}$, at which a phrase transition occurs from normal state to collapse. That is to say, when $\alpha>\alpha_{c}$, the ecoindustrial system maintains its normal and efficient functioning, while when $\alpha<\alpha_{c}, C F^{*}$ suddenly increases from 0 and cascading failure emerges because the capacity of each node is limited, propagating the whole or part network to stop working. Therefore, $\alpha_{c}$ is the least value of protection strength to avoid cascading failure, which reflects the minimum antiinterference ability of the network. Apparently, the lower the value of $\alpha_{c}$, the stronger the resilience of the network against cascading failure. The critical threshold $\alpha_{c}$ controls all the properties of the symbiosis network of an EIP and also quantifies the symbiosis network resilience by a transition from the normal state to collapse.

4.2. Theoretical Analysis. To explore the resilience against cascading failures for symbiosis networks of EIPs, some theoretical analysis is given below to discuss the critical threshold $\alpha_{c}$. Taking two cases of $\gamma=1$ and $\gamma>1$ into account, we analyze the observed cascading phenomenon on symbiosis networks. The failure probability of an overload node is denoted by $h$. Based on our removal rule of an overload node, in order to avoid the occurrence of cascading failures for a neighbor node $j$ of the removed node $i$, the following condition should be satisfied:

$$
\begin{gathered}
L_{j}+\Delta L_{i j}<C_{j}, \quad \gamma=1, \\
\frac{L_{j}+\Delta L_{i j}-C_{j}}{\gamma C_{j}-C_{j}}<h, \quad \gamma>1 .
\end{gathered}
$$

According to (1) and (3) in our cascading model, the above condition (11) can be expressed as

$$
\begin{gathered}
{\left[k_{j}^{\beta}+\left(\sum_{m \in H_{j}} k_{m}\right)^{\beta}\right]+\left[k_{i}^{\beta}+\left(\sum_{n \in H_{i}} k_{n}\right)^{\beta}\right]} \\
\times \frac{k_{j}{ }^{\beta}+\left(\sum_{m \in H_{j}} k_{m}\right)^{\beta}}{\sum_{n \in H_{i}}\left[k_{n}{ }^{\beta}+\left(\sum_{f \in H_{n}} k_{f}\right)^{\beta}\right]}
\end{gathered}
$$

$$
\begin{aligned}
& <(1+\alpha)\left[k_{j}{ }^{\beta}+\left(\sum_{m \in H_{j}} k_{m}\right)^{\beta}\right], \quad \gamma=1, \\
& \left(\left[k_{j}{ }^{\beta}+\left(\sum_{m \in H_{j}} k_{m}\right)^{\beta}\right]+\left[k_{i}^{\beta}+\left(\sum_{n \in H_{i}} k_{n}\right)^{\beta}\right]\right. \\
& \times \frac{k_{j}{ }^{\beta}+\left(\sum_{m \in H_{j}} k_{m}\right)^{\beta}}{\left.\sum_{n \in H_{i}}\left[k_{n}{ }^{\beta}+\left(\sum_{f \in H_{n}}{ }^{\beta}\right)_{f}\right)^{\beta}\right]} \\
& \times\left((\gamma-1)(1+\alpha)\left[k_{j}{ }^{\beta}+\left(\sum_{m \in H_{j}} k_{m}\right)^{\beta}\right]\right)^{-1}<h
\end{aligned}
$$

From (12), we get

$$
\begin{gathered}
1+\frac{\left[k_{i}{ }^{\beta}+\left(\sum_{n \in H_{i}} k_{n}\right)^{\beta}\right]}{\sum_{n \in H_{i}}\left[k_{n}{ }^{\beta}+\left(\sum_{f \in H_{n}} k_{f}\right)^{\beta}\right]}<1+\alpha, \quad \gamma=1, \\
\frac{1+\left[k_{i}^{\beta}+\left(\sum_{n \in H_{i}} k_{n}\right)^{\beta}\right] / \sum_{n \in H_{i}}\left[k_{n}{ }^{\beta}+\left(\sum_{f \in H_{n}} k_{f}\right)^{\beta}\right]}{h \gamma-h+1}<1+\alpha, \\
\sum_{n \in H_{i}} k_{n}{ }^{\beta}=\sum_{\tilde{k}=m}^{K} k_{i} P\left(\widetilde{k} \mid k_{i}\right) \tilde{k}^{\beta},
\end{gathered}
$$

where $m$ and $K$ are the minimum degree and the maximum degree, respectively. $P\left(\widetilde{k} \mid k_{i}\right)$ is the conditional probability that the node of degree $k_{i}$ has a neighbor of degree $\widetilde{k}$. It 
is satisfied with the normalized condition and the balance condition which is as follows:

$$
\begin{gathered}
\sum_{\widetilde{k}} P\left(\tilde{k} \mid k_{i}\right)=1 \\
k_{i} P\left(\widetilde{k} \mid k_{i}\right) P\left(k_{i}\right)=\widetilde{k} P\left(k_{i} \mid \tilde{k}\right) P(\widetilde{k}) .
\end{gathered}
$$

Based on the condition (15), equation (14) can be expressed as follows:

$$
\sum_{n \in H_{i}} k_{n}{ }^{\beta}=k_{i} \sum_{\tilde{k}=m}^{K} \frac{\tilde{k} P(\widetilde{k}) \tilde{k}^{\beta}}{\langle k\rangle}=k_{i} \frac{\left\langle k^{\beta+1}\right\rangle}{\langle k\rangle} .
$$

In the case of $\beta=1$, we get

$$
\sum_{n \in H_{i}} k_{n}=k_{i} \frac{\left\langle k^{2}\right\rangle}{\langle k\rangle} .
$$

Hence, based on (16) and (17), the condition (13) can be rewritten as follows:

$$
\begin{gathered}
1+\frac{k_{i}{ }^{\beta-1}\langle k\rangle}{k^{\beta+1}}<1+\alpha, \quad \gamma=1, \\
\frac{1+k_{i}^{\beta-1}\langle k\rangle / k^{\beta+1}}{h \gamma-h+1}<1+\alpha, \quad \gamma>1 .
\end{gathered}
$$

By the inequality (18), the critical threshold $\alpha_{c}$ can be gotten by considering the ranges of $\beta<1, \beta=1$, and $\beta>1$, respectively, as follows:

when $\gamma=1$, we have

$$
\alpha_{c}= \begin{cases}\frac{m^{\beta-1}\langle k\rangle}{\left\langle k^{\beta+1}\right\rangle}, & \beta<1, \\ \frac{\langle k\rangle}{\left\langle k^{2}\right\rangle}, & \beta=1, \\ \frac{K^{\beta-1}\langle k\rangle}{\left\langle k^{\beta+1}\right\rangle}, & \beta>1,\end{cases}
$$

and when $\gamma>1$, we have

$$
\alpha_{c}= \begin{cases}\frac{1+m^{\beta-1}\langle k\rangle /\left\langle k^{\beta+1}\right\rangle}{h \gamma-h+1}-1, & \beta<1, \\ \frac{1+\langle k\rangle /\left\langle k^{2}\right\rangle}{h \gamma-h+1}-1, & \beta=1, \\ \frac{1+K^{\beta-1}\langle k\rangle /\left\langle k^{\beta+1}\right\rangle}{h \gamma-h+1}-1, & \beta>1 .\end{cases}
$$

For (18), we consider in the case of $\beta<1$ that

$$
\begin{aligned}
\frac{m^{\beta-1}\langle k\rangle}{\left\langle k^{\beta+1}\right\rangle} & =\frac{\langle k\rangle}{(1 / N) \sum_{i=1}^{N} k_{i}^{2}\left(k_{i} / m\right)^{\beta-1}} \\
& >\frac{\langle k\rangle}{(1 / N) \sum_{i=1}^{N} k_{i}^{2}}=\frac{\langle k\rangle}{\left\langle k^{2}\right\rangle} .
\end{aligned}
$$

From the inequality (21), we find that $\alpha_{c}(\beta<1)>\alpha_{c}(\beta=$ 1) when $\gamma=1$ and $\gamma>1$. Similarly, $\alpha_{c}(\beta>1)>\alpha_{c}(\beta=1)$ when $\gamma=1$ and $\gamma>1$. Compared with other values of the parameter $\beta$, the lowest value of $\alpha_{c}$ at $\beta=1$ indicates that symbiosis systems reach the strongest resilience level against cascading failures. We know that the tunable parameter $\beta$ controls the strength of the initial load of a node. Therefore, the strongest resilience at $\beta=1$ implies an optimal initial load distribution rule, which may be useful for the EIP to avoid cascading failure triggered by production problem.

Moreover, according to (20), we find that $\alpha_{c}$ has a negative relationship with the value $\gamma$ when the value $\beta$ is given. That is to say, a bigger value $\gamma$ leads to a stronger resilience against cascading failures. The value of $\gamma$ determines to a certain extent the protection of those overload nodes. The bigger the value of the parameter $\gamma$, the higher the cost of avoiding cascading failures. This result will be very helpful for the choice on an optimal value of $\gamma$.

4.3. Numerical Simulation. The network structure plays an important role in the resilience of networks. For the symbiosis network of an EIP, if the tolerance parameter is able to be effectively selected, not only the construction cost of the ecoindustrial park will be saved, but also the overall resilience of the symbiosis network will be enhanced. Thus, many existing problems in the current planning and construction for many ecoindustrial parks will be solved. Therefore, we furthermore explore the relation between the critical threshold $\alpha_{c}$ and the other parameters in our cascading model. Figures 4-6 report the dynamical results of cascading failures in the symbiosis network of Jinjie ecoindustrial park with different values of the parameters where cascading failures are triggered by removing the nodes one by one in the set $V$.

We focus on the effect of the tolerance parameter $\alpha$ for the normalized avalanche size $C F^{*}$ for different values of $\gamma$ first. Figure 4 illustrates $C F^{*}$ after cascading failures of all removed nodes, as a function of the tolerance parameter $\alpha$ under six different values of $\gamma$ from Figures 4(a), 4(b), 4(c), 4(d), 4(e), and 4(f). From Figure 4, one can see that the value of $C F^{*}$ increases rapidly as the decrease of the tolerance parameter from 1 to 0 , which indicates that the cascading failures of symbiosis networks triggered by removing nodes can lead to serious results.

Figure 5 represents the relation between the critical threshold $\alpha_{c}$ and the tunable parameter $\beta$ for different values of $\gamma$. One can see that $\alpha_{c}$ attains the minimal value when $\beta=1$ on all the curves for different $\gamma$ in Figure 5. From Figures 4-5, we also can see an interesting phenomenon in our cascading model. The symbiosis network displays its strongest resilience against cascading failures decided by the critical threshold $\alpha_{c}$ at $\beta=1$ under six different values of $\gamma$. Therefore, the theoretical analysis result is further verified by the simulation results. These numerical simulation results agree well with the finding of theoretical analysis result. In addition, we see that the parameter $\gamma$ plays an important role in the symbiosis network resilience against cascading failures in Figures 4-5.

The effect of the parameter $\gamma$ on the critical threshold $\alpha_{c}$ is further reported in Figure 6. It is easy to find that the obtained $\alpha_{c}$ originating from the same value $\beta$ has a negative 


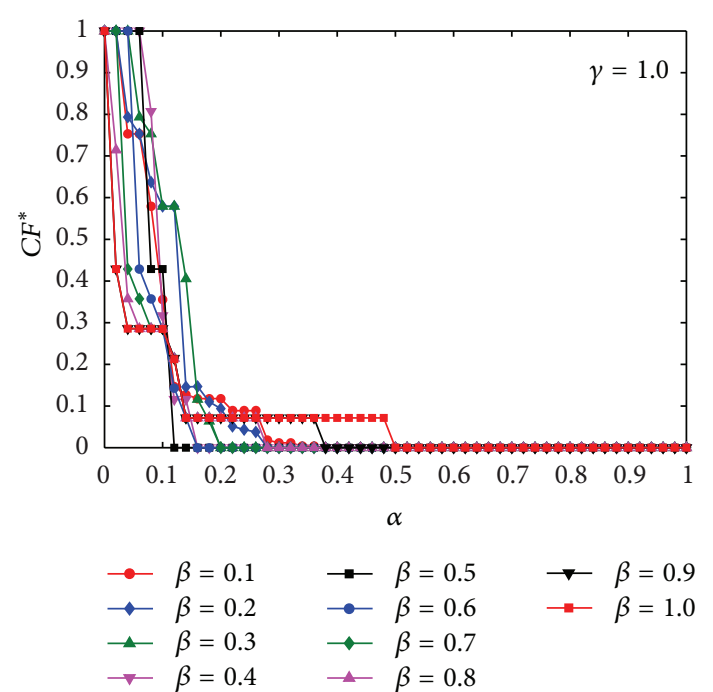

(a)

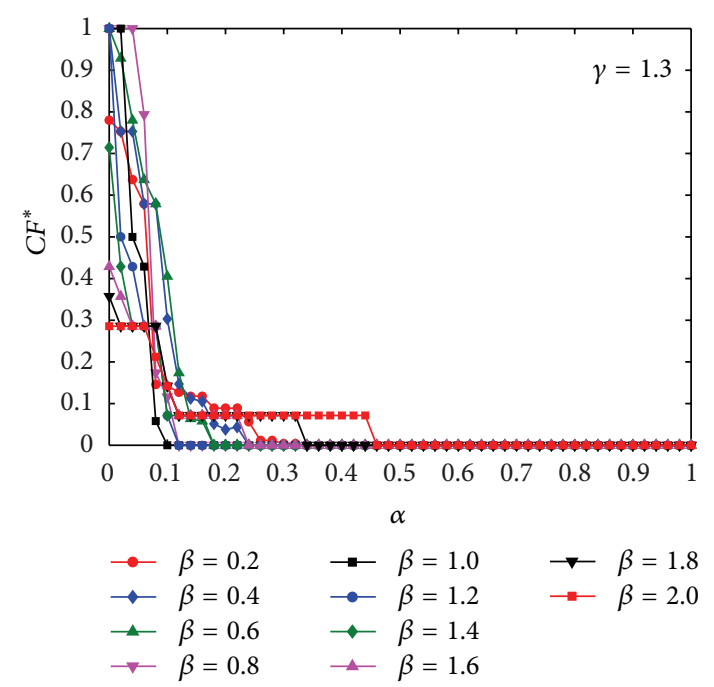

(c)

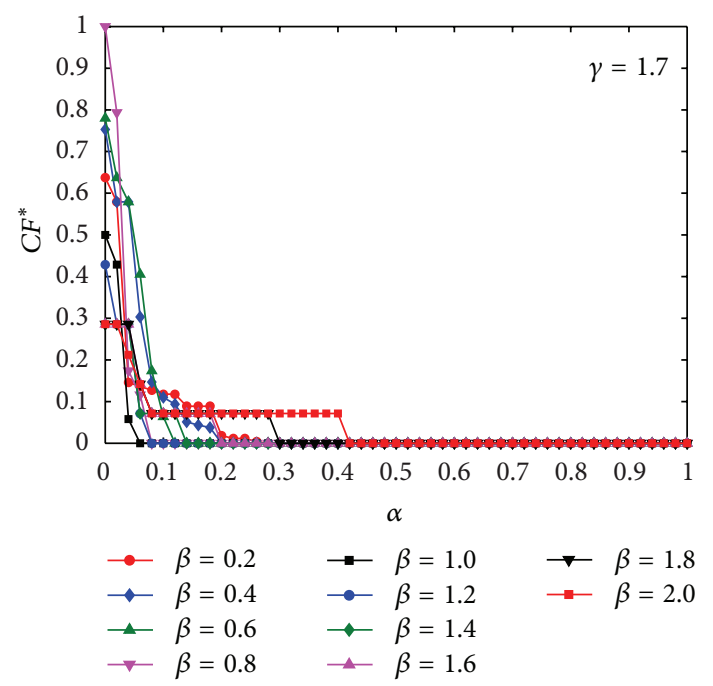

(e)

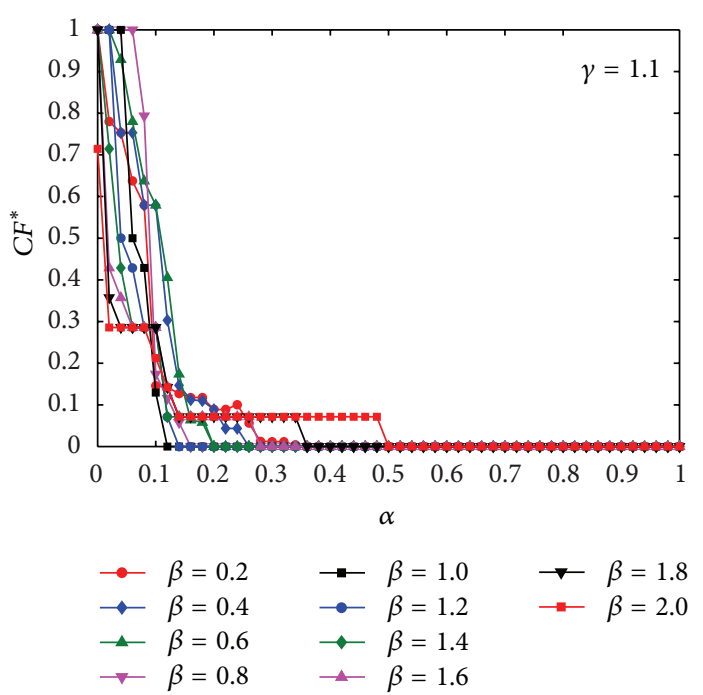

(b)

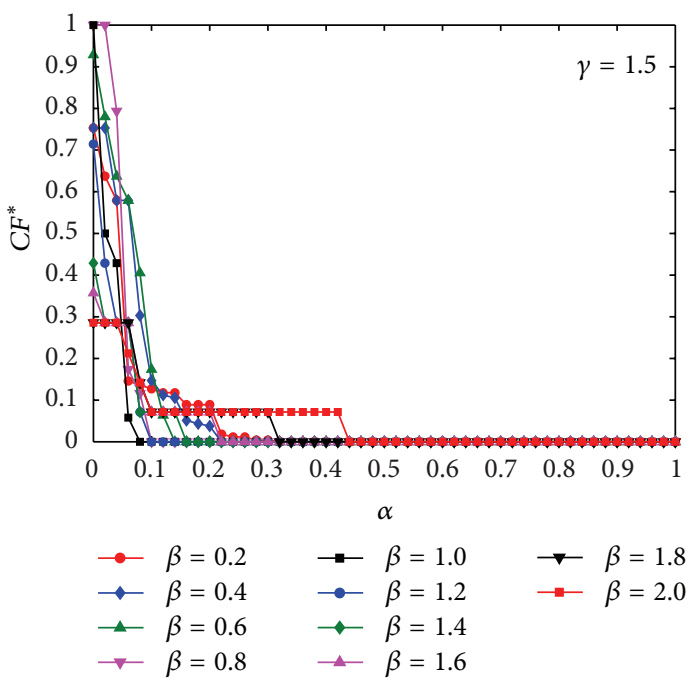

(d)

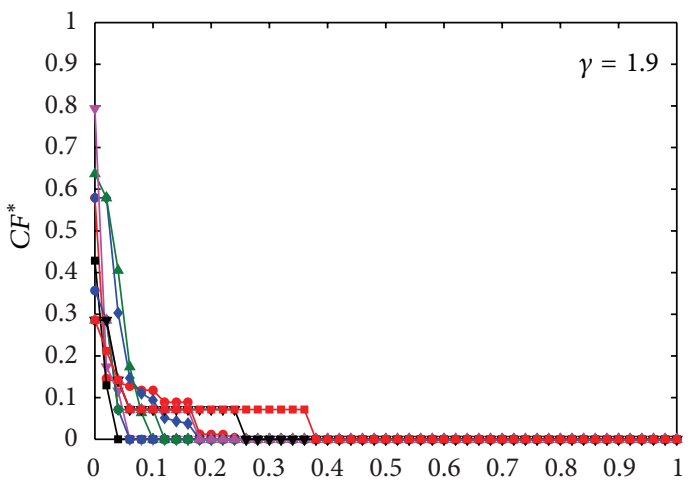

$\alpha$

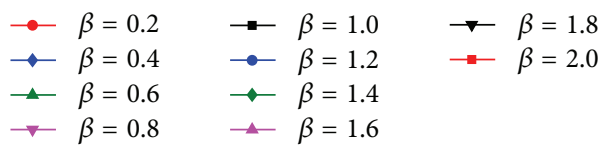

(f)

Figure 4: $C F^{*}$ as a function of the tolerance parameter $\alpha$ for different values of $\beta$. (a), (b), (c), (d), (e) and (f) show six results when $\gamma=1.0$, $1.1,1.3,1.5,1.7$, and 1.9 , respectively. 


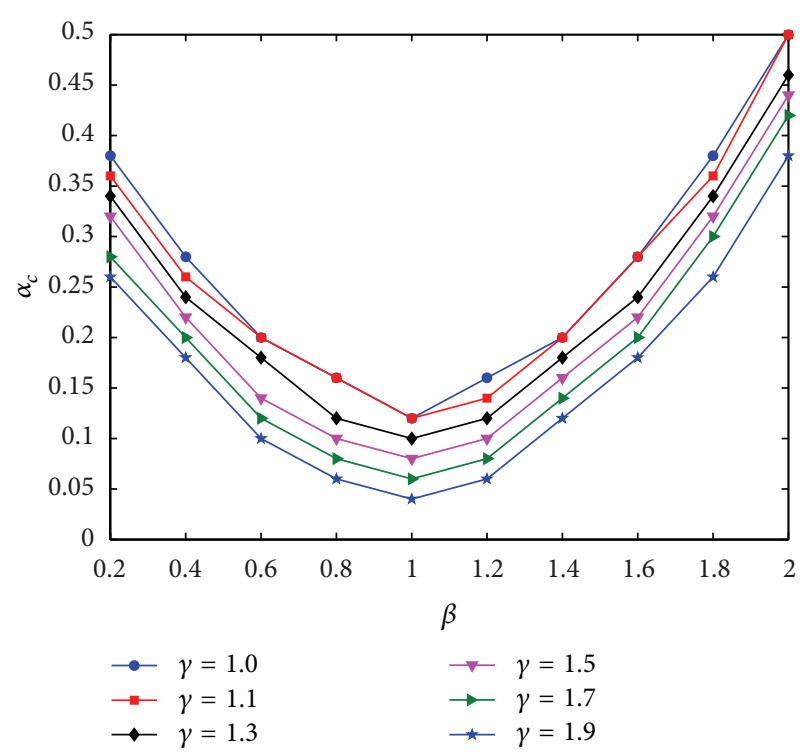

FIgURE 5: Relation between the critical threshold $\alpha_{c}$ and the tunable parameter $\beta$ for different values of $\gamma$.

relationship with the parameter $\gamma$. From Figure 6 , we can see that the curves of simulation results are in good agreement with the above theoretical analysis results and also just consistent with the actual situation. This relationship can thus supply information for monitoring and control to avoid cascading failures in the symbiosis network of Jinjie ecoindustrial park. Additionally, as is shown in Figure 6, all values on the curve $\beta=1$ are smaller than the ones on other curves in the case of the same $\gamma$, which also verify the result that the symbiosis network reach their strongest resilience level at $\beta=1$. Therefore, our model is reasonable and useful to improve the resilience of symbiosis networks of EIPs in the control and defense of the cascading failures.

\section{Conclusions}

In this paper, we construct a cascading model with tunable parameters for symbiosis networks of EIPs and explore cascading failure phenomenon in EIPs. Considering that industrial developments are usually planned to increase the recycling of wastes and by-products, we introduce the rate of ecological connectance of EIPs and propose a new evaluation method of node importance considering cascading failure according to the features of symbiosis networks of EIPs. This evaluation method can help us to find some potential critical nodes which are sensitive to the ecological degree and the functioning efficiency of networks but not so important intuitively. Furthermore, based on the proposed cascading model, we propose the critical threshold to quantify and further investigate the resilience of symbiosis networks of ecoindustrial parks. To illustrate the effectiveness and feasibility of the proposed model, we extract Jinjie ecoindustrial system in Shanxi Province of China as an undirected and unweighted symbiosis network. The cascading reaction behaviors on the symbiosis network are investigated under the cascading model.

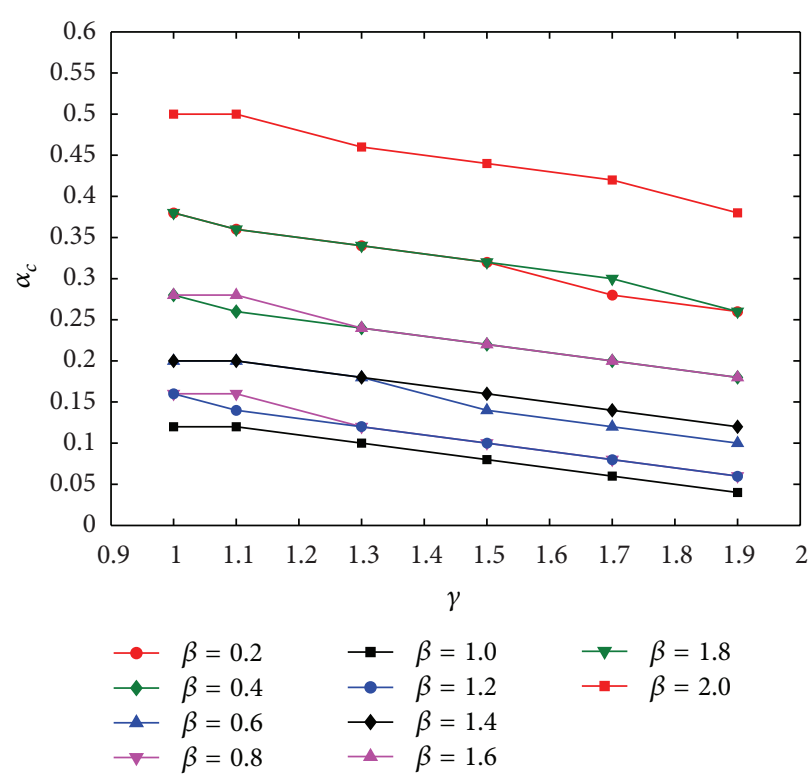

Figure 6: Relation between the critical threshold $\alpha_{c}$ and the parameter $\gamma$.

Our results show those critical nodes in the symbiosis network of Jinjie EIP. In addition, some interesting and instructive results are obtained in our proposed cascading model. The theoretical analysis results and numerical simulations both show that the symbiosis network displays the strongest resilience level against cascading failure at $\beta=1$ under the proposed cascading model. This result implies an optimal initial load distribution rule, which may be useful for the EIP to avoid cascading failure triggered by production problem. It is also seen that the critical threshold $\alpha_{c}$ has a negative relationship with the value $\gamma$. We know that the value of the tolerance parameter reflects the construction cost of an EIP. If the tolerance parameter is able to be effectively selected in construction and management of EIPs, we not only can save the construction cost of EIPs but also enhance the resilience for symbiosis networks of EIPs. It is very helpful for the construction of symbiosis networks of EIPs to select the optimal value of the parameters to attain the optimal resilience. With these results, many existing production problems in the planning and construction will be solved in EIPs.

Our work may have practical implications for protecting the key nodes effectively and avoiding cascading-failureinduced disasters in symbiosis networks. Moreover, the proposed cascading model has great generality for characterizing cascading-failure-induced disasters in the real world and provides a valuable method to many real-life networks. It is expected that the presented model may help us better understand the cascading phenomena in nature and enhance the resilience of the networks.

\section{Acknowledgment}

This work is supported by the National Natural Science Foundation of China (no. 60974076). 


\section{References}

[1] H. Li and R. B. Xiao, "A multi-agent virtual enterprise model and its simulation with Swarm," International Journal of Production Research, vol. 44, no. 9, pp. 1719-1737, 2006.

[2] S. M. Wagner and N. Neshat, "A comparison of supply chain vulnerability indices for different categories of firms," International Journal of Production Research, vol. 50, no. 11, pp. 2877-2891, 2012.

[3] S. P. Wen, Z. G. Zeng, and T. W. Huang, " $H_{\infty}$ Filtering for neutral systems with mixed delays and multiplicative noises," IEEE Transactions on Circuits and Systems Part II, vol. 59, no. 11, pp. 820-824, 2012.

[4] S. P. Wen, Z. G. Zeng, and T. W. Huang, "Robust probabilistic sampling $H_{\infty}$ output tracking control for a class of nonlinear networked systems with multiplicative noises," Journal of the Franklin Institute, vol. 350, pp. 1093-1111, 2013.

[5] S. P. Wen, Z. G. Zeng, and T. W. Huang, "Observer-based $H_{\infty}$ control of discrete time-delay systems with random communication packet losses and multiplicative noises," Applied Mathematics and Computation, vol. 219, no. 12, pp. 6484-6493, 2013.

[6] R. Cohen, K. Erez, D. Ben-Avraham, and S. Havlin, "Resilience of the Internet to random breakdowns," Physical Review Letters, vol. 85, no. 21, pp. 4626-4628, 2000.

[7] R. Cohen, K. Erez, D. Ben-Avraham, and S. Havlin, "Breakdown of the internet under intentional attack," Physical Review Letters, vol. 86, no. 16, pp. 3682-3685, 2001.

[8] A. X. C. N. Valente, A. Sarkar, and H. A. Stone, "Two-peak and three-peak optimal complex networks," Physical Review Letters, vol. 92, no. 11, 4 pages, 2004.

[9] K. Burnard and R. Bhamra, "Organisational resilience: development of a conceptual framework for organisational responses," International Journal of Production Research, vol. 49, no. 18, pp. 5581-5599, 2011.

[10] K.-I. Goh, B. Kahng, and D. Kim, "Fluctuation-driven dynamics of the Internet topology," Physical Review Letters, vol. 88, no. 10, pp. 1087011-1087014, 2002.

[11] R. Albert, I. Albert, and G. L. Nakarado, "Structural vulnerability of the North American power grid," Physical Review E, vol. 69, no. 2, Article ID 025103, 4 pages, 2004.

[12] H. Pearson, "Protecting critical assets: the interdiction median problem with fortification," Physical Review E, vol. 37, no. 19, pp. 352-367, 2004.

[13] D.-O. Leonardo, J. I. Craig, B. J. Goodno, and A. Bostrom, "Interdependent response of networked systems," Journal of Infrastructure Systems, vol. 13, no. 3, pp. 185-194, 2007.

[14] E. A. Lowe and L. K. Evans, "Industrial ecology and industrial ecosystems," Journal of Cleaner Production, vol. 3, no. 1-2, pp. 47-53, 1995.

[15] J. Korhonen, "Industrial ecology in the strategic sustainable development model: strategic applications of industrial ecology," Journal of Cleaner Production, vol. 12, no. 8-10, pp. 809823, 2004.

[16] M. Mirata and T. Emtairah, "Industrial symbiosis networks and the contribution to environmental innovation: the case of the Landskrona industrial symbiosis programme," Journal of Cleaner Production, vol. 13, no. 10-11, pp. 993-1002, 2005.

[17] A. J. P. Carr, "Choctaw Eco-Industrial Park: an ecological approach to industrial land-use planning and design," Landscape and Urban Planning, vol. 42, no. 2-4, pp. 239-257, 1998.
[18] T. Sterr and T. Ott, “The industrial region as a promising unit for eco-industrial development-reflections, practical experience and establishment of innovative instruments to support industrial ecology," Journal of Cleaner Production, vol. 12, no. 8-10, pp. 947-965, 2004.

[19] T. Graedel, B. R. Allenby, and H. Shi, Industrial Ecology, Tsinghua University Press, Beijing, China, 2004, (in Chinese).

[20] Y. M. Song and L. Shi, "Complexity measurements for Kalundborg and Gongyi industrial symbiosis networks," Journal of Tsinghua University, vol. 48, no. 9, pp. 61-64, 2008 (Chinese).

[21] R. A. Wright, R. P. Côté, J. Duffy, and J. Brazner, "Diversity and connectance in an industrial context: the case of burnside industrial park," Journal of Industrial Ecology, vol. 13, no. 4, pp. 551-564, 2009.

[22] A. Posch, "Industrial recycling networks as starting points for broader sustainability-oriented cooperation?" Journal of Industrial Ecology, vol. 14, no. 2, pp. 242-257, 2010.

[23] I. Dobson, B. A. Carreras, V. E. Lynch, and D. E. Newman, "Complex systems analysis of series of blackouts: cascading failure, critical points, and self-organization," Chaos, vol. 17, no. 2, Article ID 026103, 13 pages, 2007.

[24] J. F. Zheng, Z. Y. Gao, and X. M. Zhao, "Modeling cascading failures in congested complex networks," Physica A, vol. 385, no. 2, pp. 700-706, 2007.

[25] A. E. Motter and Y. Lai, "Cascade-based attacks on complex networks," Physical Review E, vol. 66, no. 6, Article ID 065102, 4 pages, 2002.

[26] D.-O. Leonardo and S. M. Vemuru, "Cascading failures in complex infrastructure systems," Structural Safety, vol. 31, no. 2, pp. 157-167, 2009. 


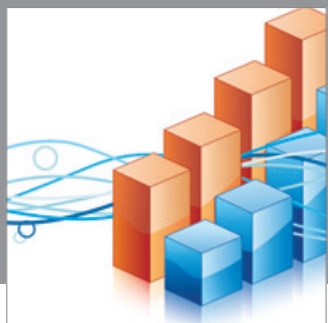

Advances in

Operations Research

mansans

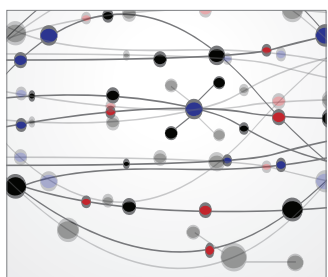

The Scientific World Journal
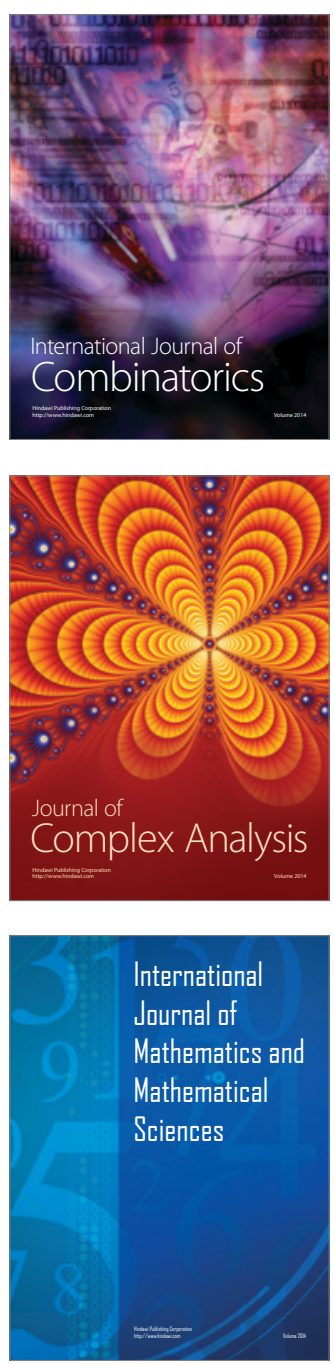
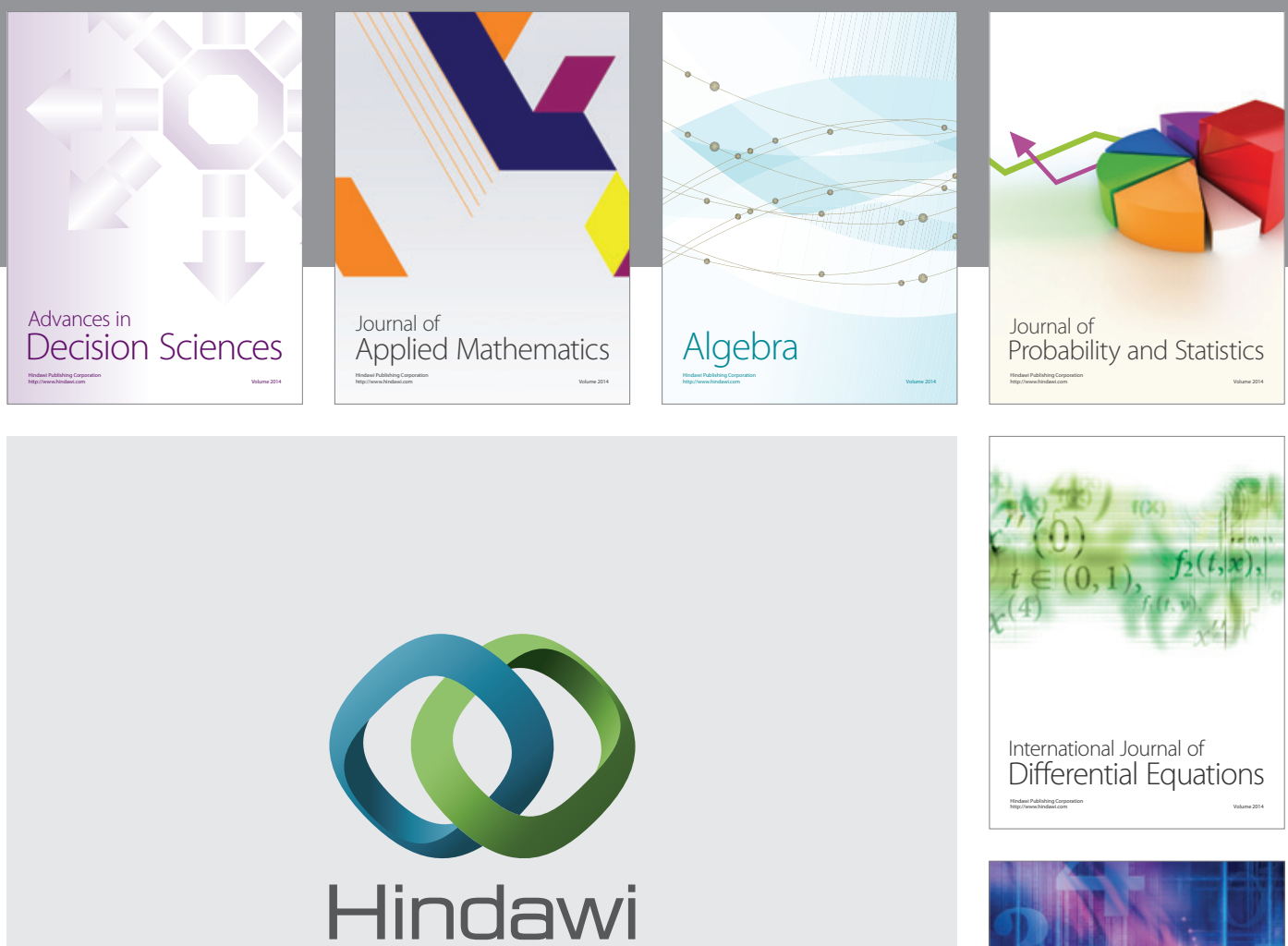

Submit your manuscripts at http://www.hindawi.com
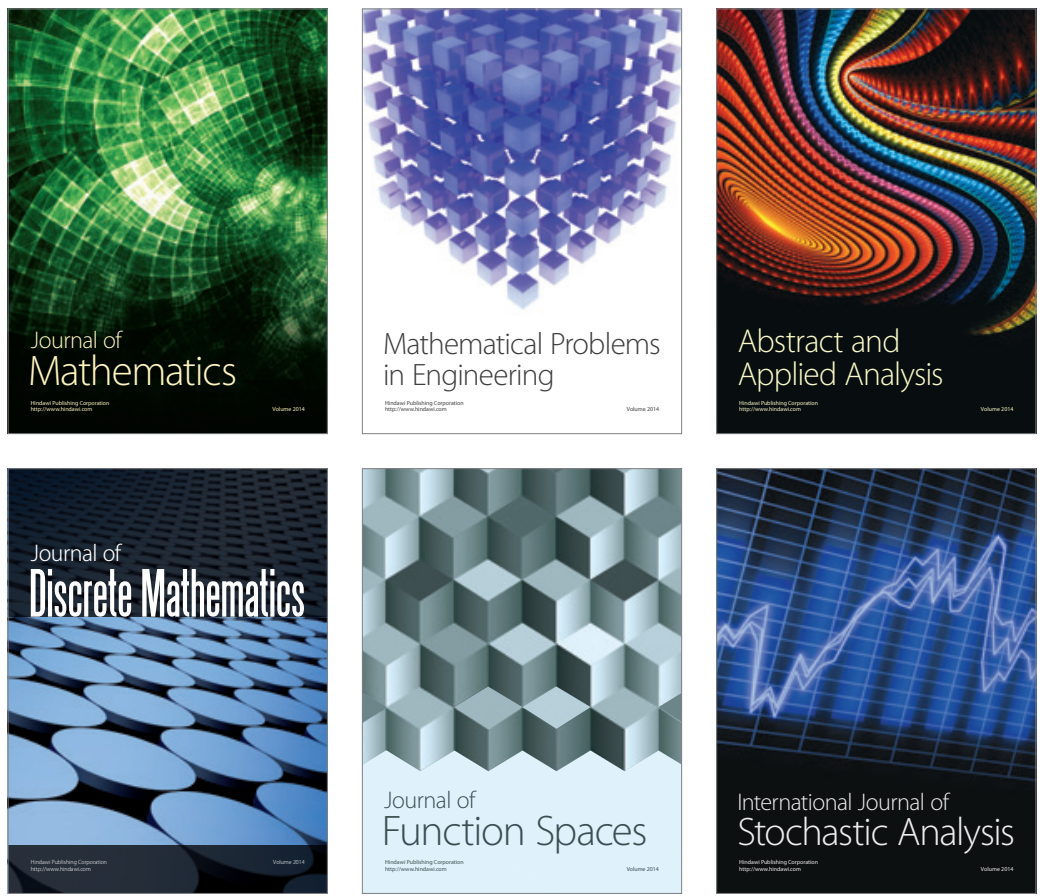

Journal of

Function Spaces

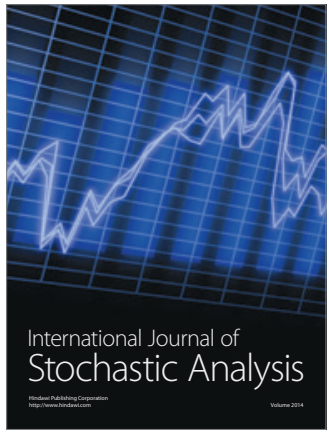

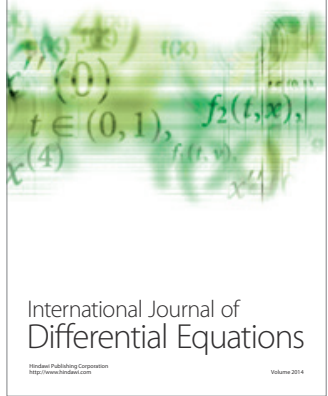
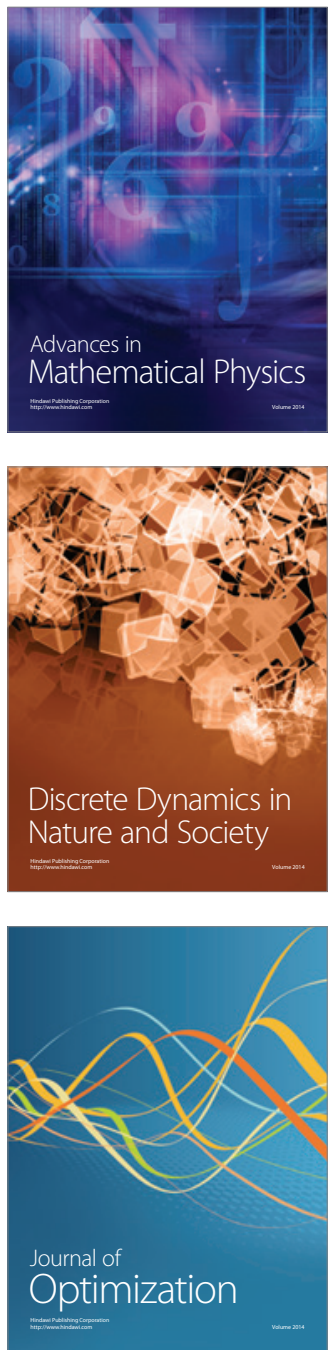